ISSN: 0213-2052 - eISSN: 2530-4100

DOI: http://dx.doi.org/10.14201/shha201937225250

\title{
DECAIMIENTO Y REACTIVACIÓN DE LA MINERÍA AURÍFERA EN EL NOROESTE PENINSULAR: LOS PROCURATORES METALLORUM Y LA ADMINISTRACIÓN ROMANA
}

\section{decay and Reactivation of Gold Mining in the Northwest of the Iberian Peninsula: Procuratores Metallorum and Roman Administration}

\author{
Narciso SANTOS YANGUAS \\ Universidad de Oviedo \\ Email: nsantos@uniovi.es
}

Fecha de recepción: 08-03-2019; aceptación definitiva: 8-5-2019

ORCID: 0000-0001-5157-4483

RESUMEN: La presencia de los procuratores metallorum como funcionarios imperiales en el marco de las explotaciones mineras de oro del Noroeste peninsular plantea numerosos interrogantes.

En primer lugar, su actuación, al parecer exclusiva, durante la segunda mitad del siglo II, tal vez como respuesta a la necesidad de una reactivación de las labores mineras en esas décadas.

Junto a ello su posible vinculación con los procuratores Asturiae et Callaeciae y, en consecuencia, con los gobernadores de la provincia Citerior Tarraconense.

Y, por último, el papel desempeñado por los beneficiarii como subalternos bajo su dirección con vistas al control y aprovechamiento de los diferentes distritos de explotación. 
Palabras clave: explotaciones mineras; Noroeste de la Península Ibérica; procuratores metallorum; beneficiarii; segunda mitad del siglo II; administración romana.

ABSTRACT: The presence of procuratores metallorum acting as imperial officers within the background of gold mining activity on the Northwest of the Iberian Peninsula raises a number of questions.

The first has to do with their activity, apparently a full-time one, during the second half of the $2^{\text {nd }}$ century, which in itself may be taken as a response to the need of reactivating mining works during those decades.

The second has to be connected with the link they might have had with theprocurators Asturiae et Callaeciae and, in turn, with the governors of the province Citerior Tarraconensis.

The third and final one has to do with the role displayed by the beneficiarii, acting as their subalterns in charge of the control of the different mining districts.

Keywords: gold mining activity; Northwest of the Iberian Peninsula; procuratores metallorum; beneficiarii; second half of the $2^{\text {nd }}$ century; Roman administration.

\section{INTRODUCCIÓN}

Hace ya una quincena de años que se llevó a cabo un estudio acerca de la importancia y significado, desde el punto de vista económico (y en cierto sentido político por su relación, connivencia y cooperación con el poder gubernamental provincial y con alguna de las unidades militares acantonadas en dicha región), de uno de estos delegados (libertos del emperador correspondiente) para los distritos mineros del Noroeste peninsular $^{1}$.

A primera vista parece deducirse que tales personajes (procuratores metallorum) dependerían en sus actividades de los procuradores identificados de forma directa con los delegados imperiales para las circunscripciones territoriales que contarían con recursos mineros susceptibles de ser aprovechados por los romanos.

En esa misma línea de investigación y al analizar la serie de funcionarios dependientes del poder imperial destinados al control y aprovechamiento de tales reservas auríferas del arco noroccidental hispano nos encontramos, en primer lugar, con la realidad de que todos estos funcionarios, de los que tenemos noticias a través de la documentación

1. SANTOS, 2004, pp. 9-33. 
PENINSULAR: LOS PROCURATORES METALLORUM Y LA ADMINISTRACIÓN ROMANA

epigráfica, corresponden en su cronología a la segunda mitad del siglo iI d. C., aunque en otras regiones ibéricas se puedan datar al parecer en fechas anteriores individuos que cumplirían tareas similares ${ }^{2}$.

Igualmente se constata que dichos delegados para la supervisión y explotación de las cortas y tajos de cada uno de los distritos mineros parecen vincularse casi en su totalidad de forma exclusiva con la región leonesa de Villalís y sus alrededores, es decir con el ámbito geográfico de Las Médulas del Bierzo y sus aledaños ${ }^{3}$, sin haber tenido conexión alguna con los distritos mineros del Norte de Portugal, configurados por los aprovechamientos de la región de Tras-os-montes, Jales, Tresminas...4, aunque tal vez, de manera tangencial al menos, pudieron haberla tenido con las explotaciones de El Caurel y la actual provincia de Lugo5.

Hemos de partir de un hecho incontestable en el desarrollo de dicho proceso económico: en el momento de poner en funcionamiento las infraestructuras técnicas (sistema hidráulico) en la explotación de los recursos mineros se hacía necesario disponer de una organización administrativa para regular dichas actividades productivas ${ }^{6}$; por lo tanto, desde la supervisión centralizada en el Estado (y, en nuestro caso, desde la propia del emperador) se configuraría a nivel administrativo una regulación (provincial, conventual y por distritos) para contribuir al laboreo intensivo de tales reservas, sobre todo auríferas ${ }^{7}$.

Así pues, la normativa edictada por la administración central, ordenada a continuación por los gobernadores provinciales y puesta definitivamente en marcha por medio de unos funcionarios específicos (procuratores imperiales), se aplicaría de manera más o menos uniforme en las diferentes zonas mineras, en las que los delegados para cada uno de los distritos del Noroeste peninsular (procuratores metallorum) y sus correspondientes subalternos (los beneficiarii, que tendrían como objetivo supervisar individualmente todo lo relacionado con cada una de las cortas o tajos),

2. Sirvan como ejemplo, los casos del liberto imperial Pudente (CIL II.956), cuya actividad se llevaría a cabo en las minas de Riotinto, o del procurator metalli Albocolensium Marco Ulpio Eutyches (CIL II.2598: I(ovi) O(ptimo) M(aximo) Anderon(i)/ sacr(um)/ M(arcus) Ulpius/Aug(usti) lib(ertus)/Eutyches) proc(urator)/metall(orum) Alboc(olensium) de la región galaica, identificable tal vez con el distrito minero de Tresminas en el norte de Portugal (aunque no sepamos a ciencia cierta si el cargo de dicho personaje en tiempos de Trajano fue realmente el de procurator metallorum o simplemente procurator de la mina (metalli) de los albocolenses. Cf. Rodríguez Colmenero, 1999, pp. 160-167.

3. Sánchez-PAlencia, 2000.

4. Ferreira de Almeida, 1973, pp. 553 ss.

5. LuZÓn, SánCheZ-PALENCia y ACuÑa, 1980.

6. Domergue, 1990 , pp. 327-340.

7. SANTOS, 2012, pp. 66 ss. 
controlaban directamente los mecanismos técnicos relacionados con la explotación de los recursos auríferos en su conjunto ${ }^{8}$.

Esta aplicación directa de la legislación imperial para el aprovechamiento intensivo de las reservas de oro traería consigo que cada uno de los distritos o centros de explotación dispusiera de su código administrativo, en el que se estipulaba lo referente a la mano de obra (que nos ofrece una marcada heterogeneidad entre los trabajadores de dichas actividades laborales ${ }^{9}$ y el mantenimiento de la estructura hidráulica en cada uno de los tajos ${ }^{10}$ ) así como lo concerniente a las campañas anuales de laboreo y al instrumental minero utilizado en el trabajo en los mismos ${ }^{11}$.

Como indicativo de este fenómeno (con unos objetivos laborales y económicos similares) se detecta en suelo de Asturias la presencia de ciertos enclaves castreños vinculados a las funciones mencionadas, uno en el conventus de los astures (San Chuis de Allande) ${ }^{12}$ y otro en el de los lucenses (el Chao Samartin, en el concejo vecino de Grandas de Salime), cuya conexión con las tareas mineras resulta evidente tanto por su ubicación en el centro de uno de los distritos más destacados del suroccidente asturiano como por los restos encontrados en el mismo, entre los que sobresale una vasija fechada a fines del siglo II d. C. ${ }^{13}$.

Además, en este contexto administrativo hemos de valorar la presencia de pequeños grupos de soldados (vexillationes) estacionados en las proximidades de los distritos mineros, contribuyendo no solo al control y mantenimiento de la infraestructura hidráulica de los mismos, sino también a su funcionamiento (dado que la mano de obra especializada estaría integrada en buena medida por miembros de estos cuerpos militares), asî

8. Hemos de tener presente la conexión que en tales actividades existiría entre estos funcionarios de la administración central y las vexillationes de la legión VII Gemina y/o de las unidades auxiliares dependientes de ella (cohorte I gálica, ala II Flavia Hispanorum civium Romanorum...).

9. Ver, por ejemplo, Mangas, 1999, pp. 245-251.

10. SÁnChez-PALENCia y SASTRE, 2002, pp. 215-234.

11. Destaca en este ámbito la elaboración de objetos de utillaje de hierro; tales funciones serían las que cumpliría, por ejemplo, el recinto castreño de El Castrelín en san Juan de Paluezas (Borrenes, El Bierzo). Cf. SÁnchez-Palencia y Fernández-Posse, 1998, pp. 227 ss.

12. SANTOS, 2009, pp. 382 ss.

13. Documento arqueológico que parece identificar dicho poblado con la Ocela mencionada en las fuentes escritas antiguas, recogiéndose en el mismo una lista de personas que, como ciudadanos, deberían aportar la carga impositiva correspondiente. Cf. VILlA, 2009, pp. 7-25. 
como al almacenamiento y salida de los productos obtenidos en las labores extractivas ${ }^{14}$.

\section{APROVECHAMIENTO DE LOS ESPACIOS MINEROS}

Los documentos en que se consigna la presencia de los procuratores metallorum en el Noroeste peninsular se corresponden en su totalidad, aunque algunos de ellos se conserven en estado fragmentado, con una de tantas manifestaciones del culto al emperador a través de la epigrafía, en este caso en el marco de la organización administrativa (y militar) romana ${ }^{15}$.

Estos monumentos, elaborados en mármol, fueron descubiertos cuando se hallaban empotrados en el ángulo suroeste de la iglesia de la localidad leonesa de Villalís de la Valduerna, perteneciente al ayuntamiento de Villamontán de la Valduerna ${ }^{16}$, habiendo sido extraídos del muro de dicho edificio religioso en el año 1968, cubiertos entonces de musgos y líquenes a pesar de que resulta evidente que dicha ubicación no correspondería a su emplazamiento originario ${ }^{17}$.

De carácter votivo, pero al mismo tiempo marcadamente administrativo, militar y económico, estas inscripciones resultan, en su conjunto, enormemente expresivas tanto por su morfología como por el contenido tan completo de su campo epigráfico, sin olvidar la iconografía de su cabecera $^{18}$.

Aunque ya Manuel Gómez Moreno las había catalogado en 1909 con motivo de su visita a esta región de la provincia leonesa con el objetivo de confeccionar su Catálogo Monumental de León, sin embargo, hasta 1968 no serían retiradas de su asentamiento correspondiente para ser depositadas en el Museo de la Real Colegiata de San Isidoro de León, donde se pueden contemplar en la actualidad.

Tales documentos se relacionan en su totalidad con la presencia de destacamentos militares (vexillationes), conectados a la legión VII Gemina (directamente o a través de ciertas unidades de tropas auxiliares

14. Ver, para el caso del distrito de Bustantigo (concejo de Allande, Asturias), GonZález, MenÉndez y Álvarez, 2008, pp. 363 ss.

15. Rabanal y García Martínez, 2001, p. 128.

16. Que se corresponde con una región eminentemente minera en tiempos romanos: DOMERGUe Y HÉrAIL, 1978.

17. GÓMez MOReno, 1979, pp. 70-71.

18. Diego Santos, 1986, pp. 51 ss. 
dependientes de ella y estacionadas en el Norte peninsular), así como con algunos oficiales que se hallaban al frente de las mismas ${ }^{19}$.

Sin embargo, el aspecto que más sobresale en todos los casos es la interrelación existente entre dichos militares y los funcionarios imperiales (procuratores metallorum) destacados en los tajos y cortas mineras de la región, así como la importancia de los funcionarios subalternos que dependían de aquellos (los beneficiarii procuratoris) y la cooperación entre todos ellos teniendo como objetivo el aprovechamiento de los recursos mineros, sin olvidar el momento en que tales personajes desempeñarían su función en el marco de las explotaciones auríferas del arco noroccidental hispano ${ }^{20}$.

Para comprender la importancia de los procuratores metallorum y de sus subalternos se hace preciso conocer, aunque sea a grandes rasgos, las características generales que rodeaban a la administración imperial de las minas aprovechadas por los romanos en territorio hispano ${ }^{21}$.

En este contexto uno de los primeros problemas se vincula con la propiedad de los terrenos susceptibles de ser aprovechados desde el punto de vista minero, lo que plantea a su vez una serie de interrogantes relacionados con las características que envolvía la propiedad del subsuelo provincial y, por tanto, de los distritos auríferos integrados en el territorio explotado por Roma y ocupado por los astures y otras poblaciones del noroeste peninsular ${ }^{22}$.

A este respecto podemos preguntarnos si se trataba de cotos de explotación en manos del Estado romano, del emperador o de particulares, y si, con vistas a su aprovechamiento, existían arrendamientos, contratas o subcontratas ${ }^{23}$.

Ninguna normativa de la legislación romana recoge los aspectos que definían las peculiaridades de la propiedad de los espacios mineros; ello derivaría del hecho de que los minerales estaban considerados como algo exclusivo del propietario del terreno o, cuando se arrendaba alguna parcela para su explotación, del usufructuario de la misma ${ }^{24}$.

19. Una participación similar del ejército parece haber tenido lugar en otras provincias del Imperio, como Moesia Superior, por ejemplo: Dusanic, 2000, pp. 343-363.

20. A este respecto resulta relevante el hecho de que los monumentos en que se mencionan dichos funcionarios imperiales se fechen todos ellos en las 4 últimas décadas del siglo II d. C.

21. Ver, entre otros, Domergue y Hérail, 1999, pp. 93-116.

22. RODRíGuez ENNES, 2002, pp. 203 ss.

23. Como sería el caso de las minas de plata de Vipasca. Cf. Orejas, 2002, pp. 255 ss.

24. En diversos pasajes del Digesto (24.3.7.13-14; 24.3.8; 7.1.13.5...) así se confirma. 
PENINSULAR: LOS PROCURATORES METALLORUM Y LA ADMINISTRACIÓN ROMANA

Sabemos que el suelo anexionado de las provincias constituía una propiedad estatal, pasando a convertirse en ager publicus o ager romanus ${ }^{25}$, lo que suponía que, tras la conquista de las comunidades indígenas su espacio geográfico pasaba a integrar la propiedad del Estado ${ }^{26}$.

Los historiadores de nuestra época coinciden en afirmar que el subsuelo de los yacimientos mineros, en el caso de minería de interior, y el suelo de los mismos, cuando se trataba de minería a cielo abierto (mucho más frecuente), correspondería al fisco imperial ${ }^{27}$ : por consiguiente los minerales pertenecían al propietario del suelo, de modo que quienes obtenían permiso de explotación no serían verdaderos propietarios, sino poseedores de unos derechos por concesión imperial durante un período de tiempo más o menos prolongado ${ }^{28}$.

Ahora bien, es posible que los antropónimos registrados en los lingotes provenientes de las labores mineras de suelo hispano se identifiquen con simples concesionarios de los aprovechamientos o con los fundidores del mineral extraído en los mismos, y no necesariamente con los poseedores de los derechos de explotación ${ }^{29}$.

La administración romana controlaría casi todas las cortas mineras, sobre todo en el caso de las reservas auríferas; de hecho, para proceder a su extracción se requería el permiso explícito del dueño del yacimiento, es decir el pueblo romano en época republicana y el emperador en la imperial ${ }^{30}$.

Por ello los distritos de explotación, que en tiempos prerromanos serían propiedad de cada una de las comunidades o de sus representantes más cualificados desde la perspectiva jerárquica (principes y magistratus de la epigrafía $)^{31}$, pasarían a ser exclusivos del Estado romano, encargado de su explotación, bien directamente bien a través de concesiones a ciudadanos privados 32 .

25. SASTRE, 2004, pp. 157 ss.

26. Ver, como ejemplo, entre otros, las explicaciones de Gayo en Instituciones 2.7. Cf. D'Ors, 1974, pp. 253 ss.

27. Domergue, 1970, pp. 163-165.

28. En contrapartida deberían cumplirse unas condiciones en relación con la conservación de la mina, el mantenimiento de la explotación... y otras, más o menos fijas, que recoge la lex metalli Vipascensis. Cf. BlázQuez, 1989, p. 120.

29. Ver, entre otros, Domergue, 1965, pp. 9 ss. 89-99.

30. Acerca de la noción romana de «espacio minero» remitimos a Domergue, 1981, pp.

31. SANTOS, 2014, pp. 547-567. 63-72.

32. Con predominio casi total del primero de dichos supuestos. Cf. Santos, 1982, pp. 
A este respecto no debemos olvidar que la reestructuración del suelo ibérico en provincias senatoriales e imperiales por el primer emperador ${ }^{33}$ implicaría que las dos circunscripciones con recursos mineros abundantes (Lusitania y Citerior) permanecieran bajo administración imperial; durante los siglos altoimperiales la explotación de dichos recursos sufriría ciertos cambios, siendo Augusto quien pondría al frente de cada una de las zonas mineras a los procuratores como funcionarios imperiales ${ }^{34}$.

Su sucesor, una vez confiscadas las minas de Sierra Morena a Sexto Mario bajo la acusación de absentismo laboral (traducido en un hipotético incesto con su hija ${ }^{35}$ las pasaría al fisco: la producción de cobre no solo era muy apreciada en tiempos del naturalista ${ }^{36}$, sino que, durante el siglo II, se exportaba en grandes cantidades al puerto de Ostia, donde era recepcionada por un procurator massae marianae, Tito Flavio Polycriso ${ }^{37}$, por lo que podemos pensar que al frente de dicha explotación se hallaba un procurator, liberto del emperador de origen griego (algo común a lo largo de la etapa altoimperial).

\section{ADMINISTRACIÓN DE LOS DISTRITOS MINEROS}

La administración romana controlaría la organización y funcionamiento de las actividades auríferas mediante los procuratores, funcionarios imperiales, divididos en dos grupos:

1. los procuratores Augusti, estructurados durante las centurias del Alto Imperio en dos clases:

- los procuratores Augusti provinciae Citerioris (bajo cuya jurisdicción se encontraba toda la provincia Citerior Tarraconense) ${ }^{38}$;

33. Cas. Dio 53.12 .

34. Aunque algunas de tales minas (como los metalla Salustianum y Livianum), continuarían en manos de particulares (Plin., N.H. 34.3-4).

35. Tac., Ann. 6.19. Ver igualmente Suet., Tib. 49.

36. Plin., N.H. 34.4.

37. CIL II.1179: T(ito) Flavio Aug(usti)/ lib(erto) Polychryso/ proc(uratori) montis/ Mariani praes/tantissumo (sic)/ confectores aeris.

38. Las funciones del primero del que tenemos noticias se fechan en el año 12-11 a. C. de nombre Q. Octavio Ser. Sagitta (A.E. 1902, n. ${ }^{\circ} 189$ = ILS 9007: Q(uintus) Octavius L(ucii) f(ilius) C(naei) n(epos) L(ucii) pronep(os) Ser(gius)/Sagitta/IIvir quinq(ennalis) III praef(ectus) fab(rum) prae(fectus) equi(tum)/ trib(unus) mil(itum) a populo procurator Caesaris/ Augusti in Vindalicis et Raetis et in valle Poe/ nina per annos III et in Hispania provincia/ per annos X et in Suria (?) biennium. 
DECAIMIENTO Y REACTIVACIÓN DE LA MINERÍA AURÍFERA EN EL NOROESTE

PENINSULAR: LOS PROCURATORES METALLORUM Y LA ADMINISTRACIÓN ROMANA

- y los procuratores Augusti Asturiae et Callaeciae, con unas tareas más definidas en el marco de la administración provincial y cuyo origen parece vincularse a la explotación intensiva de los recursos mineros del Noroeste hispano ${ }^{39}$;

2. o los procuratores metallorum, destinados a administrar, al parecer ya en una etapa avanzada, cada uno de los distritos mineros (entre otros los de Tresminas, Luyego-Teleno y Las Médulas), en cuyo caso los libertos imperiales, muchos de ellos de origen oriental $^{40}$, desempeñarían un papel fundamental ${ }^{41}$.

Relacionados con tales funcionarios estarían algunas vexillationes, a veces integradas por legionarios ${ }^{42}$ y otras por soldados de los cuerpos auxiliares dependientes de la legión VII Gemina.

Sabemos que no podría darse dependencia directa de destacamentos legionarios de un liberto, ya que, por lo general, al frente de dichas unidades se hallaba un miembro del orden ecuestre, lo que no impediría la coordinación entre el procurador y el jefe de las mismas ${ }^{43}$; estos procuradores, conectados directamente con el emperador, tendrían su residencia en la capital del conventus al que estaban asignados, es decir Braga, Lugo y/o Astorga ${ }^{44}$.

Para entender el carácter de su actividad partimos del hecho de que los distritos mineros eran monopolio del Estado; durante el Alto Imperio al identificarse el fisco con el patrimonio del emperador, los centros mineros se administrarían por este a través de un grupo de funcionarios: de esta manera los procuradores imperiales se convertirían en la máxima autoridad no solo en los aspectos administrativos sino también en los técnicos,

39. El primero del que tenemos referencias en la epigrafía (año 79 d. C.) sería Lucio Arruntio Máximo (CIL II.2477 = ILS 254: Imp(eratori) Caes(ari) Vesp(asiano) Aug(usto) pont(ifici)/ max(imo) trib(unicia) pot(estate) X imp(eratori) XX p(atri) p(atriae) co(n) s(uli) IX/ im(eratori) T(ito) Vesp(asiano) Caes(ari) Aug(usti) f(ilio) pont(ifici) trib(unicia)/ pot(estate) VIII imp(eratori) XIV co(n)s(uli) VI(I?)/

C(aio) Calpetano Rantio Quirina(le)/Val(erio) Festo leg(ato) Aug(usti) $\operatorname{pr}(o) \operatorname{pr}$ (aetore)/D(ecimo) Cornelio Maeciano leg(ato) Aug(usti)/ L(ucio) Arruntio Maximo proc(uratori) Aug(usti)/leg(ionis) VII Gem(inae) fel(icis)/ civitates X/ aquiflavienses aobrigens(es)/ bibali coelerni equaesi/ interamici limici ( $n$ )aebisoc(i)/ quarquerni tamagani).

40. García Y Bellido, 1968, pp. 191 ss.

41. Ver, por ejemplo, CIL II.2598 y BRAH 54 (1909) 27, n. 7 = ILS 9131, así como CIL II.2552-2554, aparecidos en Villalís, sobre los que volveremos más abajo.

42. Domergue, 1970 , p. 170.

43. Le Roux, 1977, pp. 365 ss. y mapa de la p. 372.

44. NONY, 1970, pp. 195 ss. 
apoyándose para dichas tareas en una serie de funcionarios de segundo nivel (procuratores metallorum y subalternos, los beneficiarios) ${ }^{45}$.

Una actividad similar desplegarían ciertos grupos de militares, que, pese a su número reducido, se identificarían con personas cualificadas desde el punto de vista técnico, de manera que su labor se focalizaría en el funcionamiento y mantenimiento del sistema hidráulico (trabajos de topografía, construcción y mantenimiento de las obras relacionadas con él ${ }^{46}$.

Sabemos que, desde los comedios del siglo I, momento en que arrancaría la fase más intensiva de aprovechamiento de los recursos mineros, Asturia-Callaecia configuraría una circunscripción financiera dentro de Hispania Citerior ${ }^{47}$ : el primer procurator para la supervisión de los distritos mineros del arco noroccidental hispano parece haber sido Lucio Arruntio Máximo, según un epígrafe de Aquae Flaviae (Chaves), fechado en el 79 d. C. ${ }^{48}$

Los registros epigráficos permiten recomponer una serie bastante completa de estos procuratores del orden ecuestre que desarrollarían su actividad en el conventus Asturum durante los dos primeros siglos de presencia romana.

Entre ellos hay que mencionar, de acuerdo con una secuencia cronológica, a Quinto Octavio Sergio Sagitta (años 12 a 3/2 a. C.) ${ }^{49}$, Quinto Licinio Silvano Graniano (época de Nerón-Galba) ${ }^{50}$, Gayo Plinio Segundo (año 73), Mario Maturo (tiempos de Vespasiano), Lucio Arruntio Máximo (año 79) $)^{51}$, Quinto Petronio Modesto (años 96-102), Julio Capitón (años 113-115), Sexto Truttedio Clemente (entre 106 y 138), Calpurnio Quadrato (entre 106 y 138), T. Furio Victorino (alrededor de 150-160), Marco Bassaeo Rufo (alrededor de 150-160), Gayo Junio Flaviano (entre 138 y 161), Publio Aelio Crispino (año 166), Flaco Aeliano (en torno a 167), Publio Cominio

45. ¿Estos últimos se hallarían en un principio a las órdenes de los procuradores imperiales y posteriormente de los procuratores metallorum, o ambos tipos de procuradores contaban con sus beneficiarii correspondientes? Cf. Sánchez-Palencia, 2002, pp. 241 ss.

46. Matías Rodríguez, 2006, pp. 5 ss.

47. SANTOS, 1982, p. 66.

48. CIL II.2477 = ILS 254 Cf. Alföldy, 1969, n. ${ }^{\circ}$ 90, p. 246. Se podría pensar que, en los años anteriores, dichos funcionarios, en cuanto a sus actividades como delegados del emperador, no se relacionarían prioritariamente con la administración minera.

49. A.E. 1902, n. $^{\circ} 189=$ ILS 9007. A pesar de que su actividad en relación con las explotaciones no parece haber sido especialmente intensa.

50. Más vinculado ya a las tareas mineras, cuyo aprovechamiento intensivo sería propiciado desde tiempos de Claudio (SANTOS, 2015, pp. 105-122), aunque ya desde los años del primer emperador romano se trataría de controlar el espacio minero correspondiente al arco noroccidental ibérico (Sánchez-Palencia y otros, 2017, pp. 863 ss.).

51. CIL II. $2477=$ ILS 254. 
DECAIMIENTO Y REACTIVACIÓN DE LA MINERÍA AURÍFERA EN EL NOROESTE

PENINSULAR: LOS PROCURATORES METALLORUM Y LA ADMINISTRACIÓN ROMANA

Clemente (años 169-170), Gayo Valerio Fusco (alrededor de 174), Publio Aelio Hilariano (de 183 a 192), Gayo Otacilio Saturnino (finales del siglo II) y Paulo Ulpio Máximo (en tiempos de Septimio Severo) ${ }^{52}$.

\section{LA FIGURA DE LOS PROCURATORES METALLORUM}

La existencia de los procuradores imperiales que tendrían a su cargo todo o gran parte del Noroeste peninsular (Asturia y Callaecia) no impediría que la autoridad principal en cada uno de los distritos (tanto en el caso de las explotaciones auríferas como argentíferas) recayera sobre el procurator metallorum en cuanto funcionario de la administración imperial, dependiente del procurador provincial correspondiente.

Esta situación se registra en la legislación de Vipasca (Aljustrel, provincia de Lusitania $)^{53}$, erigiéndose en la única normativa oficial en el territorio hispano en relación con la problemática derivada del aprovechamiento de los recursos mineros ${ }^{54}$.

Junto a los documentos epigráficos que recogen, por ejemplo, los antropónimos de los procuratores de las minas de Riotinto ${ }^{55}$, así como del procurator metalli albocolensium de un enclave galaico sin localizar (Marco Ulpio Eutyches) ${ }^{56}$, o el que se menciona en las Tablas de bronce de Vipasca (Ulpio Eliano), hay que referirse a los procuradores imperiales vinculados a las explotaciones auríferas de las cortas mineras del Noroeste peninsular.

Con respecto a su cronología resulta significativa la inscripción de Aljustrel, fechada al parecer en el último tercio del siglo II o los primeros años del siguiente, dedicada por los colonos de las minas a un procurator metallorum, que además desempeñó el cargo de vicarius rationalium ${ }^{57}$; a dichos cargos se añadiría el de restitutor metallorum, lo que parece

52. Santos, 1992, cuadro de la p. 275 . No consideramos que sea necesario un análisis pormenorizado de cada uno de estos personajes.

53. Lex metalli Vipascensis 2.4: procurator qui metallis praeerit. Cf. CAPANELLI, 1989, pp. 138 ss.

54. Más detalles acerca de la importancia y significado de estos personajes en el contexto de la administración imperial en WeAVER, 1983 (2. ${ }^{\mathrm{a}}$ edic.).

55. Como el que dedicó al emperador Nerva unas tablas de bronce (CIL II.956: Imp(eratori) Nervae Caesari Aug(usto)/ pontifici maximo tr(ibunicia) potest(ate) p(atri) p(atriae) co(n)s(uli) III/ [desi]g(nato) IIII Pudens Aug(usti) lib(ertus)/ procurator/ [de su] o posuit).

56. CIL II.2598: I(ovi) O(ptimo) M(aximo)/Anderon/sac(rum)/M(arcus) Ulpius/ Aug(usti) lib(ertus)/ Eutyches proc(urator)/ metall(i) Alboc(olensis).

57. BlázQuez, 1989, p. 123. 
relacionarse con el hecho de que las cortas de dicho distrito dejarían de aprovecharse durante un período de tiempo, quizás desde las décadas intermedias del siglo ir por motivos que desconocemos (al menos en parte), para reanudar su actividad años después, coincidiendo con la fase de desempeño de funciones de los procuratores metallorum en el Noroeste peninsular en tiempos de los últimos Antoninos. La nueva etapa de laboreo no se correspondería entonces, como se ha llegado a pensar en el caso de las minas auríferas de suelo astur, con el momento de revitalización de las tareas mineras que traería consigo la configuración de la provincia Hispania nova Citerior Antoniniana por Caracalla en el Noroeste peninsular ${ }^{58}$.

Por consiguiente, será en la segunda mitad del siglo II cuando los restos epigráficos nos permitan comprobar la presencia de una serie de procuratores metallorum, libertos imperiales, en las inscripciones de Villalís: todo lleva a identificarlos con personajes que desempeñarían la máxima autoridad en el marco de los aprovechamientos auríferos de Las Médulas durante las últimas décadas de dicha centuria, ejerciendo las funciones de legados del poder imperial, aunque quizás a un segundo nivel ${ }^{59}$.

De tales personajes dependientes del poder imperial, vinculados a destacamentos militares romanos (legionarios o de tropas auxiliares) con los que coparticiparían en las labores mineras, la documentación epigráfica permite conocer, por orden cronológico, a los siguientes:

- Hermes, Augustorum libertus, que desarrollaría su actividad alrededor del año $163^{60}$;

58. Diego Santos, 1974 , pp. 472-474.

59. Sobre la morfología, estructura y contenido del campo epigráfico de tales documentos ver Rabanal y García MarTínez, 2001, pp. 126 ss.

60. CIL II.2552 = ILS 9125 y A.E. 1910, n. ${ }^{\circ}$ 3: I(ovi) O(ptimo) M(aximo)/ [pr]o salute M(arci) Aureli(i) An/ [t]onini et L(ucii) Aureli(i) Veri/ [Aug/ustor(um) ob natale(m) aq[ui/l] ae vexillatio leg(ionis) VII G(eminae) [F(elicis)]/ sub cura Licini(i) Pater[ni]/ c(enturionis) leg(ionis) eiusd(em) et Hermetis/ Augustor(um) lib(erti) proc(uratoris) et Lu/ creti(i) Paterni dec(urionis) coh(ortis)/ I Celt(iberorum) et Fabi(i) Marcian[i]/ b(ene)f(iciarii) proc(uratoris) Augustor(um) et/[IJuli(i) Iuliani sign(iferi) leg(ionis)/ eiusd(em). IIII id(us) Iunias/ Laeliano et Pastore co(n)[s(ulibus)] (10 de junio del año 163). Cf. GarCía y Bellido, 1966, p. 32.

Ver igualmente CIL II.2555: I(ovi) O(ptimo) M(aximo)/pro salute M(arci) Aureli(i)/ Antonini et L(ucii) Aureli(i) Veri/ Augustorum ob natale(m)[aprunculorum?]/ milites coh(ortis) I Gall[i]c(ae)/ sub cura [H]e[r]met(is) Aug[ustor(um) lib(erti)]/ [p]r[oc(uratoris)] et Lucret[i(i)] Paterni/ [dec(urionis)] coh(ortis) [I Celtib(erorum)] et Ful[---]/---- Cf. LE Roux, 1972, p. 134, n. $^{\circ} 28$. 
DECAIMIENTO Y REACTIVACIÓN DE LA MINERÍA AURÍFERA EN EL NOROESTE

PENINSULAR: LOS PROCURATORES METALLORUM Y LA ADMINISTRACIÓN ROMANA

- seguidamente desempeñaría unas funciones similares Zoilus, Augustorum libertus, al menos entre 165 y $167^{61}$;

- a continuación lo haría Aurelius Eutyches, Augusti libertus, en torno a los años 181 a $184^{62}$;

- y, por último, Aurelius Firmus, Augusti libertus procurator metallorum, que se hallaría al frente de uno de estos distritos en el año 191, tratándose del único que se menciona expresamente con el cargo completo ${ }^{63}$.

Respecto a este último extremo consideramos que no sería preciso que aparecieran en los monumentos epigráficos todos ellos con el título

61. CIL II.2556 = ILS $9129=$ A.E. 1910, n. ${ }^{\circ}$ 6: [I(ovi) O(ptimo) $M$ (aximo)/ pro salute M(arci) Aureli(i)]/ Anton[ini et L(ucii) Aureli(i)]/Ve[ri Augustor(um) o]b n[atale(m)]/ apr[unculor(um)] millites]/ coh(ortis) I Galll(icae) sub c]ur[a Zo/ illi Augustor(um) lib(erti) pr[oc(uratoris)/ et] Val(erii) Flavi(i) c(enturionis) coh(ortis) ei[us/ delm et Val(erii) Valentis b(ene)f(iciarii)/[pro]c(uratoris) August[or(um) et] Iulli(i)/Iul]iani sign(iferi) leg(ionis) VII [G(eminae)] F(elicis)/X K(alendas) Maias Pud[ente et Pollione/ co(n)s(ulibus)]. (22 de abril del año 165). Cf. García y Bellido, 1966, p. 33.

Este mismo personaje aparece reflejado en una inscripción encontrada igualmente en Villalís de la Valduerna (CIL II.2553 = ILS 9127): I(ovi) O(ptimo) M(aximo)/pro salute M(arci) Aureli(i) Antonin[i]/ et L(ucii) Aureli(i) Veri Augustorum/ ob natale(m) signor(um) vexillatio/ coh(ortis) I Celtib(erorum) sub cura Zoili Augustor(um) lib(erti) proc(uratoris) et Val(erii) Flavi(i)/ c(enturionis) coh(ortis) I Gall(icae) et Aeli(i) Flavi(i) b(ene)f(iciarii) proc(uratoris)/ Augustor(um) et Lucreti(i) Mater/ ni imag(iniferi) leg(ionis) VII G(eminae) F(elicis) et Iuli(i) Sel duli(i) tesserari(i) c(obortis) I C(eltiberorum) posital idib(us) octobrib(us) impe(ratore) Aurel lio Vero III et Quadrato co(n)s(ulibus) (15 de octubre del año 167).

62. La primera fecha aparece confirmada por una inscripción de Luyego de Somoza (García y Bellido, 1966, pp. 26-27 = A.E. 1967, n. ${ }^{\circ} 230: I$ (ovi) O(ptimo) M(aximo)/pro salute M(arci) Aureli(i)/Commodi Aug(usti) ob/ natale(m) aquil(a)e lelg(ionis)]/VII G(eminae) F(elicis) sub cura [M(arci)]/ Aur(elii) Eutychetis Alug(usti)/ lib(erti)] proc(uratoris) et M(arci) Mes/ [solris c(enturionis) coh(ortis) I Galli/ [ca]e et Aviti Paterni/ [de]c(urionis) Augusto/ et Bur(r)o co(n)s(ulibus) (10 de junio del año 181) y la segunda por CIL II.2554 = ILS 9126: I(ovi) O(ptimo) M(aximo)/ pro salute M(arci) Aureli(i) Co/ mmodi Antonini Aug(usti)/ ob natale(m) aqui/ lae vexillatio/ leg(ionis) VII G(eminae) F(elicis) sub clu]/ ra Aur(elii) Eutych[et(is)]/ Aug(usti) lib(erti) proc(uratoris) et VIal(erii)]/ Semproniani d[ec(urionis)]/ alae II Flaviae IIII i[d(us)]/ iun(ias) Marullio et Aeliano co(n)s(ulibus) (10 de junio del año 184).

Para más detalles remitimos a Diego Santos, 1986, n. ${ }^{\circ} 35$, pp. 53-55.

63. ILS $9131=$ A.E. 1910, n. ${ }^{\circ}$ 2: II(ovi) O(ptimo) M(aximo)/pro salute M(arci) Aure/ li(i) Commodi An/tolnini Pii Fel(icis) Aulg(usti)/Gelr(manici) Max(imi) trib(unicia) pot(estate) [XVI]/ [i]mp(eratoris) XV co(n)s(ulis) VI ob n[a]/ tale (m) aprunculoru[m]/ mil(ites) coh(ortis) I Gal(licae) sub culra]/ Aureli(i) Firmi Aug(usti) lib(erti) [proc(uratoris)]/ met(allorum) et Valer(ii) Marcel[lini]/ dec(urionis) al(ae) II Fl(aviae) [X] K(alendas) Mali(as)] Popilio Pedone et [B]radua Mauriclo co(n)s(ulibus)] (22 de abril del año 191). Cf. VIGIL, 1961, p. 107. 
de procurator metallorum dado que la similitud que hallamos en dichos documentos, junto con el desempeño de dicho cargo y la presencia de las vexillationes correspondientes, nos lleva a pensar que desempeñarían, en el ámbito minero, unas actividades similares.

En cuanto a su antroponimia nos permite afirmar que la mayoría de ellos tendrían origen oriental, lo que explicaría que en un conjunto de inscripciones descubiertas en Asturica y Legio (o aledaños), en las que se mencionan procuratores imperiales, estos fueran devotos de dioses orientales $^{64}$.

A este respecto entre los procuradores imperiales de clase ecuestre, de acuerdo con un orden cronológico paralelo al expuesto en el caso de los procuratores metallorum del Norte peninsular (y hasta el momento del cese de las explotaciones en dicha circunscripción geográfica) tenemos noticias de los siguientes:

- Publio Aelio Crispino (año 166);

- Flaco Aeliano (en torno al 167);

- Publio Cominio Clemente (años 169-170);

- Gayo Valerio Fusco (ca. 174);

- Publio Aelio Hilariano (entre los años 183 y 192) ${ }^{65}$;

- Gayo Otacilio Octavio Saturnino (ca. 192 a 198) ${ }^{66}$;

- Julio Silvano Melanión, procurator Augustorum provinciae Hispaniae Citerioris entre 198 y $209^{67}$, quien cumpliría con anterioridad funciones similares en la región de Dalmacia (en concreto en el enclave minero del Municipium Domavianum), donde se registra en un documento epigráfico como procurator argentariorum ${ }^{68}$, lo

64. García y Bellido, 1968, pp. 191 ss. Cf. igualmente Nony, 1970, pp. 195 ss.

65. A.E. 1968, n. ${ }^{\circ}$ 228: Iovi Optimo Maximo/ Iunoni Reginae/ Minervae Victricil P(ublius) Ael(ius) P(ublii) f(ilius) Hilarianus/proc(urator) Aug(usti) cum liberis/ pro salute [[M(arci) Aur(elii) Commodi Antonini]] Aug(usti)/ Pii Fel(icis) [[ ]].

Ver igualmente A.E. 1968, n. ${ }^{\circ}$ 227: Di(i)s deabusque/ quos ius fasque est/ precari in Pantheo/ P(ublius) Ael(ius) P(ubli) f(ilius) Hilarianus/proc(urator) Aug(usti) cum liberis/ pro salute [[M(arci) Aur(elii) Commodi Antonini]]/ Aug(usti) [[Pii Fel(icis)........]]. Cf. Diego SANTOS, 1986, pp. 24-25 y 26-27 respectivamente.

66. A.E. 1968, n. ${ }^{\circ} 234=$ ILER 5960: Fortunae Reduci sanctael G(aius) Otacilius Octavius/Saturninus v(ir) e(gregius) proc(urator)/Aug(usti)/ dicavit cum filia et/ nepote. Cf. García y Bellido, 1968, p. 206 y Nony, 1970, p. 199.

67. CIL II.1729 y 1732: I(ovi) O(ptimo) M(aximo)/Custodi/ Iunoni Reginae/ Minervae Sanctae/ ceterisque dis/ deabusque/ inmortalibus/Iulius Silvanus/ Melanio proc(urator)/ Aug(ustorum)/provinc(iae) Hisp(aniae) Citer(ioris)/ dicavit. Cf. Diego SAnTos, 1968, pp. 97-98 y $98-99$.

68. HiRT, 2010, p. 160, nota 277. 
DECAIMIENTO Y REACTIVACIÓN DE LA MINERÍA AURÍFERA EN EL NOROESTE

PENINSULAR: LOS PROCURATORES METALLORUM Y LA ADMINISTRACIÓN ROMANA

que parece indicar que los procuradores imperiales especializados en la administración de los distritos mineros serían desplazados comúnmente de una zona minera a otra ${ }^{69}$;

- y, por último, Publio Ulpio Máximo, cuyas tareas administrativas parecen conectarse ya con la época de Septimio Severo ${ }^{70}$.

Según ciertos investigadores habría que añadir a este colectivo 2 funcionarios imperiales más:

- Saturninus, Augusti libertus ${ }^{71}$, cuyas tareas administrativoeconómicas vinculadas a la minería del Noroeste peninsular corresponderían a las décadas finales del siglo $\mathrm{II}^{72}$;

- y Claudio Zenobio, también de origen oriental, cuyas actividades se llevarían a cabo entre 212 y $222^{73}$, coincidiendo con la reactivación laboral en el Noroeste tras el impulso recibido de Caracalla.

\section{FUNCIONARIOS SUBALTERNOS DE LOS PROCURADORES}

De la misma manera esos textos epigráficos, cuya cronología corresponde mayoritariamente a tiempos de Marco Aurelio, documentan los antropónimos de un conjunto de beneficiarii procuratoris Augusti, cuya tarea, en el contexto de la administración minera, estribaría, en tanto que funcionarios subalternos, en actuar como contables de uno de tales procuratores ecuestres ${ }^{74}$.

Como en el caso de sus superiores administrativo-mineros nos referiremos por orden cronológico a los que tenemos constatada su existencia, fechados en la segunda mitad del siglo II d. C.:

69. Marcos, 1986, pp. 219 ss.

70. A.E. 1968, n. ${ }^{\circ}$ 233: Fortunael bonae Reduci/ P(ublius) Ul(pius) Maximus/ proc(urator) Aug(usti)/ cum uxore/ et filio. Cf. Diego SANTos, 1986, n. ${ }^{\circ}$ 9, p. 28 (la lectura que realiza no creemos que sea la correcta).

71. Le Roux, 1976, p. 87: [I(ovi) O(ptimo) M(aximo)/numi]ni/b(us) Augu]sto(rum)/ [Iunoni RJelgilnae/ Veneri Victrici/ Africae Caelesti/ Frugifero/ (Genio coloniae) Augustae Emeritae/ et Larib(us) Gallaeciar(um)/Saturninus Aug(usti) lib(ertus). Cf. Canto, 1979, pp. 301-306.

72. Christol y Demougin, 1990, pp. 159 ss

73. BlÁZquez, 1989, p. 123.

74. ¿Dependerían igualmente de los procuratores metallorum, quienes a su vez lo harían de los procuradores imperiales? 
- Fabio Marciano, beneficiarius procuratoris Augustorum en el 163 según una inscripción de Villalís ya analizada ${ }^{75}$, en que se le asocia con el procurator Hermes;

- poco después Elio Flavio, beneficiarius procuratoris Augustorum en el 167 de acuerdo con otro documento epigráfico de Villalís ${ }^{76}$, asociado al parecer con el procurator Zoilus;

- y, por último, algunos años después (en el 175) Valerio Semproniano, beneficiarius procuratoris Augusti, de acuerdo con otro epígrafe de la misma localidad leonesa ${ }^{77}$, sin mencionar al procurator al que debería acompañar ${ }^{78}$.

La presencia de los beneficiarii en los mismos documentos epigráficos que los procuratores y su mención a continuación de los mismos ha llevado a pensar que estarían a sus órdenes, aunque en realidad ningún testimonio notifica que dependiesen directamente de los procuratores metallorum y no de los procuradores imperiales.

Sabemos que la administración minera en las restantes provincias imperiales que disponían de estos mismos recursos (Dacia sobre todo) sería similar a lo que sucedería en el Noroeste hispano, de manera que tanto los sistemas técnicos para el funcionamiento de las minas de oro como los mecanismos de la regulación administrativa serían similares ${ }^{79}$.

Hechos comparables se constatan en las zonas mineralizables de las provincias danubianas ${ }^{80}$, o en los centros mineros del Norte de África (Egipto), Grecia, Siria, Britannia y Galia: en todos ellos actuarían procuratores imperiales, al igual que procuratores metallorum, al frente de los distritos durante los siglos del Alto Imperio ${ }^{81}$.

75. CIL II.2552 = ILS 9125. Cf. LE Roux, 1972, p. 134, n. ${ }^{\circ} 28$.

76. CIL II.2553 = ILS 9127. Cf. GarCía y Bellido, 1959, p. 32.

77. A.E. 1910 , n. $^{\circ} 1$ = ILS 9130: I(ovi) O(ptimo) M(aximo) s(acrum)/ pro salute imp(eratoris)/Caes(aris) M(arci) Aur(elii) Anto/ nini Aug(usti) ob natalle(m)]/ aprunculorum/ milites coh(ortis) I Gal(licae)/ sub cura M(arci) Senti(i)/ Bucconis )(centurionis) coh(ortis) eiusdem/ et Val(erii) Sempronian/i]/ beneficiari( $i$ ) proc(uratoris) Aug(usti)/X $k$ (alendas) Mai(as) Pisone et Iuliano co(n)s(ulibus). Cf. García y Bellido, 1959, p. 35, fig. 11.

78. Este personaje aparece algunos años después, en concreto en el 184, como decurión del ala II Flavia, de acuerdo con una inscripción hallada igualmente en Villalís (CIL II. $2554=$ ILS 9126).

79. Ver, por ejemplo, Dusanic, 1989, pp. 148 ss.

80. Para más detalles ver, entre otros, Dusanic, 1977, pp. 52 ss. En alguna inscripción se recoge incluso el calificativo de procurator metallorum Pannonicorum et Dalmaticorum.

81. Una explicación más amplia se encuentra, por ejemplo, en Blázquez, 1989, pp. 123-125. 
PENINSULAR: LOS PROCURATORES METALLORUM Y LA ADMINISTRACIÓN ROMANA

Vamos a tratar de comprender, a partir de aquí, el significado de los procuratores metallorum y de los beneficiarii, a quienes podemos considerar como sus ayudantes subalternos: la presencia en la circunscripción territorial de Asturia-Callaecia de un procurator imperial, encargado de los tres conventus jurídicos del Noroeste, tal vez halle explicación en relación con el acuartelamiento de la legión VII Gemina en León, puesto que descubrimos en este hecho una connivencia y coparticipación entre el elemento administrativo y el militar; en este sentido el geógrafo de Amasia documenta que en su época (la de Augusto, y concretamente tras el final de las guerras astur-cántabras), los procuratores ecuestres tendrían como misión distribuir el dinero de la paga de los soldados ${ }^{82}$.

Desconocemos, sin embargo, el momento de implantación de dicha normativa: partimos de que los cuerpos de ejército que participarían en la anexión del suelo astur estaban integrados por varias legiones y unidades de tropas auxiliares ${ }^{83}$, hallándose entre ellas las que configurarían después las tropas ocupación en el Norte peninsular (legiones IIII Macedonica, VI Victrix y X Gemina $)^{84}$.

En el transcurso de los combates de las guerras astur-cántabras Publio Carisio dispondría de las legiones VI Victrix y X Gemina; al finalizar los enfrentamientos la VI se asentaría en el suelo que pasaría a ser capital del conventus de los lucenses (Lucus Augusti) ${ }^{85}$, mientras que la X establecería su campamento en el futuro centro político-administrativo de los astures

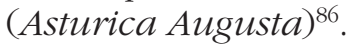

La actuación de un procurator para recaudar los impuestos de las 3 circunscripciones territoriales del Noroeste peninsular, mediante los que se podría suministrar a los militares estacionados en la zona (ejército de ocupación) la paga para su subsistencia y soldada, constituiría una simplificación de tesorería, acorde con los intereses de la administración romana ${ }^{87}$.

82. Strab. 3.4.20.

83. Los detalles acerca de sus nombres, componentes y documentos que corroboran su presencia en el Norte peninsular aparecen recogidos en SANTOS, 2016, pp. 83-127.

84. SANTOS, 1993, pp. 165 ss.

85. RODRÍGueZ COLMENERO, 1997, pp. 7 ss.

86. GonZÁlez, 1998, pp. 1019 ss.

87. Bajo el mando del procurator Asturiae et Callaeciae, posiblemente ya desde una etapa temprana, a pesar de que el aprovechamiento intensivo de los recursos auríferos haya que llevarlo a los comedios del siglo I, existirían procuratores de rango inferior, sin que sepamos si en realidad se trataba de procuratores metallorum y beneficiarii, cuyas funciones se asemejarían a las desempeñadas por los que aparecen en la documentación epigráfica de la segunda mitad del siglo il d. C. Cf. Mangas y Orejas, 1999, pp. 220 ss. 
Ciertos investigadores del siglo xx consideraban que este procurator no sería más que un subordinado del procurator de la Hispania Citerior ${ }^{88}$, lo que corroboraría el testimonio estraboniano; se trataría del más destacado de la serie de procuratores que dependían, en grado muy diverso, del gobernador provincial ${ }^{89}$, ya que, por medio de él, se ejercía la autoridad, en materia financiera y en los aspectos administrativo-judiciales, en Asturia-Callaecia.

Posiblemente las condiciones especiales que habían generado el surgimiento de un procurator para dicho territorio servirían de base para el origen, en la segunda década del siglo III, de una nueva circunscripción provincial, que comprendía todo el Noroeste peninsular, aunque no contase más que con una existencia reducida en el tiempo: en ese marco se comprende la creación (y rápida desaparición) de Hispania nova Citerior Antoniniana en el ámbito de la administración provincial de Caracalla ${ }^{90}$.

Se buscaría así, como objetivo prioritario, aunque no exclusivo, la reactivación de la explotación aurífera a través de una organización administrativo-territorial más compleja, así como de una supervisión más directa sobre cada uno de los distritos mineros ${ }^{91}$.

No obstante, dado que los resultados no alcanzarían un nivel económico satisfactorio, al no obtenerse de dicha revitalización la rentabilidad deseada, este intento final por intensificar la obtención de oro sería inútil, dándose paso a un debilitamiento de dichas actividades y a la desaparición, casi inmediata, de la nueva provincia; a este respecto no debemos olvidar que los procuratores de rango ecuestre de Asturia y Callaecia dejan de aparecer en la documentación epigráfica alrededor del $222^{92}$, coincidiendo precisamente con la etapa de reducción en las actividades auríferas del cuadrante noroccidental.

88. Albertini, 1923, pp. 52 ss.

89. Este personaje pasaría el invierno en Tarraco o Cartago Nova, mientras que durante los meses estivales recorrería y controlaría todo lo relacionado con el buen funcionamiento (político-administrativo, económico-social y religioso) de la provincia que tenía encomendada.

90. Santos, 1983, pp. 47 ss. Cf. Diego Santos, 2009, pp. 659 ss.

91. CIL II.2661 = ILS 1157 y 5680: [I(ovi) O(ptimo) M(aximo)]/ Iunoni Relginae]/ pro salute et im[peri(i)]/ diuturnitalte imp(eratoris)]/ M(arci) Aurelii Anto[nini]/ Pii Fel(icis) Aug(usti) et Iulliae]/ Piae Fel(icis) Aug(ustae) maltris]/ Antonini Aug(usti) [cas]/ trorum senat[us]/ ac patriae/ C(aius) Iul(ius) Cerealis co(n)s(ul) [leg(atus)]/Aug(usti) pr(o) pr(aetore) pr(ovinciae) H(ispaniae) n(ovae) C(iterioris) An[toni]/ nianae post divi[sam]/ provinc(iam) primus ab eo [mi(ssus)]. Ver igualmente A.E. 1929, n. ${ }^{\circ} 158$.

92. El último en desempeñar este cargo pudo haber sido Julio Silvano Melanión (CIL II.1729 y 1732 = BRAH 163 (1968) 191 ss., n. ${ }^{\text {os }} 1,2$ y 3) o Claudio Zenobio ya en tiempos de Caracalla (cf. BlázQuez, 1989, p. 123). 
PENINSULAR: LOS PROCURATORES METALLORUM Y LA ADMINISTRACIÓN ROMANA

Puesto que la producción de las cortas mineras estaba controlada por el emperador mediante este funcionario, quien contaría bajo su jurisdicción con todo el Noroeste peninsular y se serviría, para supervisar los distritos de explotación, de los procuratores metallorum, apoyados en los beneficiarii como subalternos para la supervisión y control de sus tareas mineras (al frente de cada una de las cortas o tajos), las actividades correspondientes a ambos se debilitarían con el paso del tiempo.

Y en consecuencia, se pondría fin a sus actividades desde los años finales del siglo II d. C., de modo que la revitalización de las labores mineras emprendida por Caracalla no contaría ya con la participación de estos delegados imperiales; es más, Gayo Julio Cereal, primer y posiblemente único gobernador de la Hispania nova Citerior Antoniniana con capital en León (ejemplo de la conjunción de intereses y funciones entre administración y ejército), sería elegido gobernador de la nueva provincia y supervisor de las antiguas tareas de los procuratores metallorum como ayudantes del procurator imperial.

\section{Conclusiones}

Con respecto a la figura de los procuratores metallorum como funcionarios imperiales encargados de las actividades laborales vinculadas al aprovechamiento de los recursos auríferos en el Noroeste peninsular se plantea toda una serie de cuestiones, y algo parecido sucede en relación con sus subalternos (al menos teóricamente), los beneficiarii procuratoris.

En primer término, sobresale el problema derivado de la época de aparición de los mismos y su incidencia en el proceso productivo supervisado por la administración romana: ¿sus funciones, conectadas con los centros mineros, arrancan ya de tiempos de Augusto de acuerdo con lo que parece deducirse del inicio y mantenimiento de otras explotaciones mineras en diversas regiones del suelo hispano desde las décadas iniciales del Imperio?

Bien es verdad que tenemos constancia de la existencia de procuratores en el Mediodía peninsular coincidiendo con los tiempos de los primeros emperadores romanos, aunque no se especifica que se encontrasen personalmente al frente de la dirección de la explotación de los recursos auríferos y/o argentíferos, a pesar de que controlasen la manipulación posterior de dichos productos en dirección a la capital del Imperio (es el caso del liberto imperial Pudente en las minas de Riotinto).

Por otro lado, desconocemos si su labor se reduciría de forma exclusiva a los centros mineros de oro o se ampliaría igualmente a los de 
plata, como sucedería con la explotación minera de Aljustrel (Lusitania) de acuerdo con lo que se deduce de la lex metalli Vipascensis, aun cuando en ciertos casos se tratase de arrendamientos imperiales a particulares (ya en el siglo II de nuestra era).

La documentación antigua (epigrafía de la región leonesa) únicamente hace mención, a través de media docena de monumentos, a 4 personajes que desempeñarían el cargo de procurator metallorum, a pesar de que solo Aurelio Firmo se registra con el título completo de su cargo en el 191 d. C.

Tal vez habría que incluir aquí al liberto imperial Marco Ulpio Eutyches, al parecer de tiempos de Trajano, si en realidad el documento epigráfico en que aparece reseñado, descubierto en suelo galaico (aunque para otros investigadores pertenecería a territorio vacceo) permite una reconstrucción como proc(urator) metall(orum) en lugar de proc(urator) met(alli) Alboc(olensium).

En el encabezamiento de las inscripciones halladas en León descubrimos la dedicatoria a Júpiter y a la salud (pro salute) del emperador correspondiente frente a los documentos de los procuratores de rango superior en los que se mencionan otras divinidades, orientales en ocasiones.

Por lo que concierne a la cronología de dichos personajes sabemos que sus actividades administrativas en el arco noroccidental hispano únicamente se fechan en la segunda mitad del siglo II, lo que nos lleva a preguntarnos por los motivos que incidirían en ello: a primera vista parece lógico pensar que, a partir de la década de los 60 de dicha centuria, tendría lugar una reactivación o intensificación en la producción, bien porque, desde los comedios de dicho siglo, se habría producido un decaimiento en la explotación, bien porque se necesitaba obtener una aportación mayor de dinero (oro y/o plata) para subvencionar algunas actividades encabezadas por el poder imperial, como el enfrentamiento con las poblaciones germanas en el limes renano-danubiano, a cuyo frente se encontraban Marco Aurelio y Cómodo.

Igualmente puede resultar significativo el hecho de que la desaparición de dichos funcionarios, de acuerdo con la documentación epigráfica, se produjese justamente en los momentos inmediatamente posteriores a la dinastía de los Antoninos, asistiendo durante un par de décadas a un cierto abandono de las explotaciones durante los primeros emperadores Severos hasta que Caracalla recurra de nuevo a su revitalización, tal vez como consecuencia de las nuevas necesidades económicas y financieras del Estado romano.

Como resultado de ello la actividad desplegada por los procuratores metallorum durante las 4 últimas décadas del siglo II, buscando una 
PENINSULAR: LOS PROCURATORES METALLORUM Y LA ADMINISTRACIÓN ROMANA

reactivación de las labores mineras, pudo constituir un precedente de lo que con posterioridad sería el intento de intensificación de dichas tareas por el mencionado emperador con la configuración de la provincia Hispania nova Citerior Antoniniana.

Otro problema se vincula con el marco geográfico que abarcaban los distritos mineros sobre los que dichos funcionarios pudieron actuar: ¿se trataría exclusivamente del espacio habitado por los astures augustanos, es decir el correspondiente a Las Médulas del Bierzo y a las explotaciones de sus alrededores, o más bien a todas las cortas y tajos incluidos en el Noroeste peninsular?

Y, junto a ello, surge una nueva cuestión: ¿por qué motivo no ha aparecido hasta la fecha rastro alguno de los mismos en el espacio minero romano del suroccidente de Asturias? Quizás la respuesta tenga que ver con el hecho de que estarían agotados ya en esos momentos los recursos auríferos desde el punto de vista de la rentabilidad de los tajos.

A este respecto podemos pensar que distritos como Naraval-NavelgasParedes (concejos de Tineo y Valdés), Bustantigo, la sierra del Palo, Montefurado... (concejo de Allande), explotaciones de Cangas del Narcea (Monterroso...) habrían sido abandonados con anterioridad y no se programaría una revitalización de los mismos hasta la actuación de Caracalla, si es que realmente el impulso de este emperador incidió sobre dicho marco geográfico, o más bien las cortas de explotación se hallaban ya clausuradas.

En este contexto surge un nuevo interrogante: ¿por qué se produce la desaparición de estos legados imperiales coincidiendo con el final de los Antoninos, es decir en la fecha que marca el paso de la muerte de Cómodo a ese año convulso de enfrentamientos internos que desembocaría en la implantación de la nueva dinastía con Septimio Severo?

Y, por otro lado, ¿es posible que se diese paso a cambios sustanciales desde el punto de vista administrativo-territorial, e incluso financiero, en las provincias occidentales, especialmente en los territorios implicados en el aprovechamiento de los recursos auríferos, es decir la provincia Citerior Tarraconense y, por paralelismo, la Dacia?

¿O bien con la nueva dinastía en el poder tendría lugar un debilitamiento acusado en las actividades mineras, que únicamente se compensaría, al menos parcialmente y con vigencia temporal escasa, con el impulso propuesto por Caracalla, a pesar de que no alcanzase demasiado éxito ni se prolongase durante muchos años?

En el caso de los beneficiarii se acumulan igualmente numerosos interrogantes: ¿desde un primer momento constituye un grupo de funcionarios subalternos vinculados directamente a los procuratores metallorum como subordinados en el Norte peninsular ibérico?; ¿o, por el contrario, 
dependerían de los procuradores imperiales, cuyo campo de actuación sería mucho más amplio que el correspondiente a estos procuratores metallorum?

La implicación de los beneficiarii en la explotación de los diferentes tajos y cortas mineros es lo que parece deducirse de los monumentos epigráficos de Villalís, donde se les asocia, en una actividad conjunta, con los procuratores y algunos militares, bien de la legión VII Gemina bien de algunas de las unidades de tropas auxiliares dependientes de ella.

Pero ¿qué sucede con anterioridad? No existe documentación de la que se deduzca la presencia de tales funcionarios antes de las 4 últimas décadas del siglo iI: en la epigrafía solo se mencionan 2 de dichos personajes vinculados con procuratores metallorum, a saber, Fabio Marciano, asociado al procurator Hermes (año 163) y Aelio Flavio, vinculado al procurator Zoilus (año 167), teniendo que añadir a Valerio Semproniano (año 175), a pesar de que desconozcamos el nombre del procurator con el que podría relacionarse.

Tales circunstancias nos llevan a preguntarnos si los beneficiarii reemplazaban en sus funciones a los procuratores metallorum o serían ellos, en última instancia, los encargados de ejecutar y cumplir los objetivos programados en el marco del proceso de producción en cada uno de los distritos.

¿Estos mismos beneficiarios pudieron ser los supervisores directos del aprovechamiento de algunos de los distritos mineros, en especial de los de dimensiones más reducidas? ¿Y de ahí que en la inscripción que se fecha en el año 175 se registre únicamente al beneficiario Valerio Semproniano sin alusión alguna al procurator metallorum al que, teóricamente al menos, estaría asociado? ¿O, por el contrario, dependerían en realidad directamente del procurador imperial y no del procurador de las minas?

En cualquier caso parece haber existido un triple nivel en el marco de la administración minera del Noroeste peninsular: los procuradores imperiales del orden ecuestre, que dependerían a su vez del gobernador provincial de la Citerior Tarraconense y que formarían parte del entramado económico vinculado al erario imperial desde tiempos de Augusto, tendrían bajo sus órdenes a los procuratores metallorum, tal vez surgidos ya en la segunda mitad del siglo II como complemento para un aprovechamiento más intensivo de los recursos mineros, en especial auríferos.

Finalmente, en la base de esa situación económica relacionada con los aprovechamientos mineros se hallarían los beneficiarii como funcionarios subalternos dependientes directamente de los procuratores metallorum, dado que su ámbito geográfico y temporal se relaciona precisamente con el que estos parecen haber controlado. 
PENINSULAR: LOS PROCURATORES METALLORUM Y LA ADMINISTRACIÓN ROMANA

A pesar de todo es posible que, en última instancia, pudieran recibir igualmente órdenes de los procuradores imperiales, encargados del control administrativo y económico del Noroeste peninsular y por ello calificados en ocasiones como procuratores Asturiae et Callaeciae.

\section{Bibliografía}

AlBerTinI, E.: Les divisions administratives de l'Espagne romaine. Paris, De Boccard, 1923.

AlföLDY, G.: Fasti Hispanienses. Wiesbaden Franz Steiner, 1969.

Blázquez, J. M.: "Administración de las minas en época romana. Su evolución", en Minería y metalurgia en las antiguas civilizaciones mediterráneas y europeas. Madrid Dirección General de Bellas Artes y Archivos, 1989, 2, pp. 119-132.

CANTO, A. M. ${ }^{a}$ : «Saturninus, Augusti libertus», Gallaecia, 3-4, 1979, pp. 301306.

CAPANELLI, D.: "Aspetti dell'amministrazione mineraria iberica nell'età del Principato", en Minería y metalurgia en las antiguas civilizaciones mediterráneas y europeas. Madrid Dirección General de Bellas Artes y Archivos, 1989, 2, pp. 138-147.

Christol, M. y Demougin, S.: "De Lugo à Pergame: la carrière de l'affranchi Saturninus dans l'administration impériale", MEFRA, 202, 1990, pp. 159-211.

Diego Santos, F.: "Ocho lápidas votivas de Astorga”, Archivum, 18, 1968, pp. 91-106.

Diego Santos, F.: «Provincia Hispania nova Citerior Antoniniana d'après deux inscriptions de León (CIL II.2661 y 5680)», en Actas del VI Congreso Internacional de Epigrafía Griega y Latina. München, 1974, pp. 472-474.

Diego SANTOS, F.: Inscripciones romanas de la provincia de León. León Diputación Provincial (CSIC), 1986.

Diego SANTOS, F.: "Nuevas consideraciones sobre la provincia Hispania nova Citerior Antoniniana», en El conventus Asturum y anotaciones al Noroeste hispano. Oviedo KRK, 2009, pp. 659-667.

Domergue, C.: "Les Planii et leur activité industrielle en Espagne sous la République", $M C V, 1,1965$, pp. 9-27.

Domergue, C.: "Les exploitations aurifères du Nord-ouest de la Péninsule Ibérique sous la domination romaine», en La minería hispana e iberoamericana. León Diputación Provincial, 1970, 1, pp.151-193.

Domergue, C.: "La notion d'espace minier dans l'antiquité gréco-romaine», Pallas, 28, 1981, pp. 89-99. 
Domergue, C.: Les mines de la Péninsule Ibérique dans l'Antiquité romaine. Collection de l'École française de Rome, 1990.

Domergue, C. y Hérail, G.: Mines d'or romaines d'Espagne. Le district de la Valduerna (León). Toulouse Université de Toulouse-le Mirail, 1978.

Domergue, C. y Hérail, G.: "Conditions de gisements et exploitation antique à Las Médulas (León-Espagne)", en L'or dans l'Antiquité. Toulouse Université de Toulouse-le Mirail, 1999, pp. 93-116.

D’Ors, A.: "La condición jurídica del suelo en las provincias de Hispania», en Atti del convegno intern. Sul tema: I diritti nelle province romane con particolare risguardo alle condizioni giuridiche del suolo. Roma 1974, pp. 253-268.

Dusanic, S.: "Aspects of Roman Mining in Noricum, Pannonia, Dalmatia and Moesia Superior", $A N R W$ 2.1, 1977, pp. 52-94.

Dusanic, S.: «The Roman Mines of Illyricum: Organization and Impact on Provincial Life,, en Minería y metalurgia en las antiguas civilizaciones mediterráneas y europeas. Madrid Dirección General de Bellas Artes y Archivos, 1989, 2, pp. 148-156.

DusANIC, S.: "Army and Mining in Moesia Superior", en Gedenkschrift E. Birley. Stuttgart Franz Steiner Verlag, 2000, pp. 343-363.

Ferreira De Almeida, C. A.: "Aspectos da mineraçao romana de ouro en Jales e Tresminas (Tras-os-montes)», en XX CAN. Zaragoza 1973, pp. 553-562.

García y Bellido, A.: "Cohors I Gallica equitata civium Romanorum", Conimbriga 1, 1959, pp. 29-40.

García y Bellido, A.: "Nuevos documentos militares de la Hispania romana", AEA, 39, 1966, pp. 24-40.

García y Bellido, A.: "Lápidas votivas a deidades exóticas halladas recientemente en Astorga y León", BRAH, 163, 1968, pp. 191-209.

Gómez Moreno, M.: Catálogo monumental de España. Provincia de León (1906-1908). Madrid, 1925 (ed. facsímil 1979).

González, M. ${ }^{a}$ L.: "Las defensas campamentales de Asturica Augusta", en Los orígenes de la ciudad en el Noroeste hispánico. Lugo, Diputación Provincial, 1998, pp. 1019-1038.

González, D.; Menéndez, A. y Álvarez, V.: «El campamento de Moyapán (Ayande, Asturias)", en Congreso de Arqueologia de Vilalba = Férvedes, 5, 2008, pp. 363-371.

HiRT, A. M.: Imperial Mines and Quarries in the Roman World. Organization Aspects 27 BC-AD 235. Oxford University Press, 2010.

Le Roux, P.: "Recherches sur les centurions de la legio VII Gemina", MCV, 8, 1972, pp. 89-146. 
PENINSULAR: LOS PROCURATORES METALLORUM Y LA ADMINISTRACIÓN ROMANA

Le Roux, P.: "Lucus Augusti, capitale administrative au Haut-Empire", en Actas del bimilenario de Lugo. Lugo, Diputación Provincial, 1976, pp. 83-106.

LE Roux, P.: "L'armée de la Péninsule Ibérique et la vie économique sous le Haut Empire romain", en Armées et fiscalité dans le monde antique. Paris, CNRS, 1977, pp. 341-372.

Luzón, J. M., SÁnchez-Palencia, F. J. y Acuña, F.: El Caurel. Madrid, Ministerio de Cultura, 1980.

Mangas, J.: "Los obligados por operae en las minas de Vipasca (Aljustrel, Portugal)», Pallas, 50, 1999, pp. 245-251.

Mangas, J. y Orejas, A.: "El trabajo de las minas en la Hispania romana", en El trabajo en la Hispania romana. Madrid, Sílex, 1999, pp. 207-313.

Marcos, M. A.: "Estudio prosopográfico del procurador de Asturica Augusta C. Iulius Silvanus Melanio", en I Congreso Internacional Astorga Romana. Astorga, Ayuntamiento, 1986, pp. 219-225.

Matías Rodríguez, R.: "La red hidráulica de Las Médulas: trazado e ingeniería", Estudios bercianos, 30-31, 2006, pp. 5-86.

Nony, D.: "Á propos des nouveaux procurateurs d'Astorga", AEA, 43, 1970, pp. 195-201.

Orejas, A.: "Aspectos técnicos y organizativos del trabajo en la lex metalli Vipascensis", Artifex. Ingeniería romana en Hispania. Madrid, Ministerio de Educación, Cultura y Deporte, 2002, pp. 255-272.

RABANAL, M. A. y GARCía MARTínez, S.: Epigrafía romana de la provincia de León: revisión y actualización. León, Universidad, 2001.

Rodríguez Colmenero, A. (coord.): Lucus Augusti. El amanecer de una ciudad. A Coruña, Diputación Provincial, 1997.

Rodríguez COLMENERO, A.: «¿Resulta factible identificar la región minera de Tres Minas, norte de Portugal, con el Metallum Albucrarense de Plinio?", $M D A I(M)$, 40, 1999, pp. 160-167.

RODRíguez EnNES, L.: "Consideraciones en torno al marco jurídico de la minería hispano-romana", Dereito: Revista xuridica da Universidade de Santiago de Compostela, 11, 2002, pp. 203-219.

SÁnchez-PALENCIA, F. J.: "La red hidráulica en las minas romanas de oro del noroeste hispano: las Médulas", en Artifex. Ingeniería romana en Hispania. Madrid, Ministerio de Educación, Cultura y Deporte, 2002, pp. 241-254.

SÁnchez-PAlencia, F. J. (coord.): Las Médulas (León): un paisaje cultural en la Asturia Augustana. León, Diputación Provincial (Instituto Leonés de Cultura), 2000.

SÁnchez-Palencia, F. J. y Fernández-Posse, M. ${ }^{\text {a }}$ D.: "El beneficio del oro para las comunidades del noroeste peninsular", en Minerales y metales en 
la prebistoria reciente: algunos testimonios de su explotación y laboreo en la Península Ibérica. Valladolid, Universidad, 1998, pp. 227-246.

Sánchez-Palencia, F. J. y SAstre, I.: "La red hidráulica de las minas de oro hispanas: aspectos jurídicos, administrativos y políticos", $A E A, 75$, 2002, pp. 215-234.

SÁNCHEZ-PALENCIA, F. J. y otros: "Augusto y el control administrativo y territorial de las zonas mineras del Noroeste hispano", Gerión, 35, 2017 (ejemplar dedicado a La Hispania de Augusto), pp. 863-874.

SANTOS, J.: "Formas de propiedad y producción en el Noroeste hispánico en época romana altoimperial", $M H A, 3,1982$, pp. 63-72.

SANTOS, N.: "La provincia Hispania nova Citerior Antoniniana", Boletín Brigantium, 4, 1983, pp. 47-60.

Santos, N.: La romanización de Asturias. Madrid, Istmo, 1992.

SANTOS, N.: «Proceso de conquista y ejército romano de ocupación en el Norte peninsular", en Indígenas y romanos en el Norte de la Península Ibérica. San Sebastián, 1993, pp. 165-179.

SANTOS, N.: «Una inscripción de Villalís (León): los procuratores metallorum y la administración minera romana en territorio de los astures", Astorica, 23, 2004, pp. 9-33.

SANTOS, N.: Asturias, los astures y la administración romana durante el Alto Imperio. Oviedo, KRK, 2009.

SANTOS, N.: Hábitat castreño, minería del oro y romanización del suroccidente de Asturias: el concejo de Allande. Oviedo, KRK, 2012.

SANTOS, N.: "Elites locales en la Asturias romana: principes y magistratus", en De Roma a las provincias: las elites como instrumento de proyección de Roma. Córdoba, Universidad, 2014, pp. 547-567.

SANTOS, N.: «El emperador Claudio y las minas de oro romanas del Noroeste de la Península Ibérica", HAnt, 39, 2015, pp. 105-122.

SANTOS, N.: Los astures y el ejército: militares y civiles en época romana. Madrid, Ministerio de Defensa, 2016.

SASTRE, I.: "Ager publicus y deditio: reflexiones sobre los procesos de provincialización", en Hommages à Monique Clavel-Levêque. Besançon Université de Franche-Comté, 2004, pp. 157-192.

VIGIL, M.: "Ala II Flavia Hispanorum civium Romanorum", AEA, 34, 1961, pp. 104-113.

VILLA, A.: «¿De aldea fortificada a Caput Civitatis? Tradición y ruptura en una comunidad castreña del siglo I d. C.: el poblado de Chao Samartín (Grandas de Salime, Asturias)», CuPAUAM, 35, 2009, pp. 7-25.

Weaver, R. C.: Familia Caesaris. A Social Study of the Emperor's Freedmen and Slaves. Cambridge University Press, 1983 (2. ${ }^{a}$ edic.). 